Soumis à Mécaniques \& Industries

Septembre 2000

\title{
Modélisation du comportement et de la rupture des matériaux sous sollicitations dynamiques
}

\author{
Modelling deformation and failure \\ of materials under dynamic loading conditions.
}

André Dragon ${ }^{1}$, François Hild ${ }^{2 *}$, Laurent $\operatorname{Rota}^{3}$ et Hervé Trumel ${ }^{1}$

\footnotetext{
${ }^{1}$ Laboratoire de Mécanique et de Physique des Matériaux (UMR $\mathrm{n}^{\circ} 6617$ CNRS-ENSMA) Ecole Nationale Supérieure de Mécanique et d'Aérotechnique, BP 40109, Téléport 2, 1 avenue Clément Ader, F-86961 Futuroscope Chasseneuil.

${ }^{2}$ LMT-Cachan (ENS Cachan-UMR n ${ }^{\circ} 8535$ CNRS-Université Paris VI), 61 avenue du Président Wilson, F-94235 Cachan Cedex.

${ }^{3}$ DGA/Centre Technique d'Arcueil/Département MSP 16bis avenue Prieur de la Côte d'Or, F-94114 Arcueil Cedex.
}

*Correspondant : Tél. : 01474021 92, Fax : 01474022 40, Mèl : hild@,lmt.ens-cachan.fr. 


\title{
Modélisation du comportement et de la rupture des matériaux sous sollicitations dynamiques
}

\author{
Modelling deformation and failure \\ of materials under dynamic loading conditions.
}

André Dragon, François Hild, Laurent Rota et Hervé Trumel

Résumé :

On assiste à un accroissement notable de la demande d'analyses (thermo-)mécaniques sous sollicitations rapides pour lesquelles les méthodes usuelles sont souvent mal adaptées. Une brève analyse permet de dégager divers régimes dynamiques en fonction de l'importance des effets inertiels. On montre également que la classique notion d'échelle spatiale devient variable selon les échelles temporelles considérées. Dans de nombreuses situations, les outils expérimentaux, théoriques et numériques sont en développement, et n'ont pas atteint un degré de maturité satisfaisant. Ceci d'autant plus que la nature dynamique des phénomènes ajoute plusieurs degrés de complexité à leur analyse : propagation des ondes, échauffement et influence de la température sur le comportement, phénoménologie dépendant de la vitesse de sollicitation, hétérogénéité des chargements, nature spatio-temporelle des échelles caractéristiques, sont autant d'effets à considérer. Un rapide bilan des connaissances permet d'identifier les manques dans les domaines de l'expérimentation, du développement de modèles de comportement et de dégradation, et des outils numériques correspondants. On constate que, selon les matériaux, cet état de l'art apparaît assez disparate, les matériaux « récents » (polymères, composites, mousses) sont les moins favorisés, même si les métaux, bétons, céramiques, verres, etc., nécessitent eux aussi de nombreux approfondissements.

Les axes principaux de développements futurs sont dégagés et ont fait l'objet de la création d'un groupe de travail commun des associations MECAMAT et DYMAT, membres de l'Association Française de Mécanique. Sa première réunion de travail s'est tenue à l'ENSMA Poitiers, les 4 et 5 avril 2000.

Mots clés : déformation / endommagement / rupture / matériaux / chargements dynamiques 


\begin{abstract}
:
There is an increasing demand for thermomechanical computations of structures under dynamic loading conditions for which the current methods are often unsatisfactory. A simple analysis quickly shows that different dynamic regimes can be distinguished depending on the magnitude of inertia effects. Furthermore, it is shown that the space scaling becomes time-dependent. Experimental, theoretical and numerical tools are still evolving and have not yet reached a sufficient degree of maturity. This is due to the inherent complexity of dynamic problems such as wave propagation, heating and thermomechanical coupling, rate-dependent phenomenology, space- and time-dependent characteristic scales. An overview of the current knowledge allows one to identify missing developments in experiments, constitutive models including degradation and failure effects, as well as computational strategies. It is shown that the state of the art is very disparate when considering different material classes such as polymers, composites and foams for which few studies are conducted. For metals, concrete, ceramics and glasses some results are available but more advanced studies are still needed.
\end{abstract}

Some future directions of research are sketched and they constitute the scope of the newly created working group common to the MECAMAT and DYMAT societies, both members of the French Society of Mechanics. Its first meeting was held April 4-5, 2000 in Poitiers.

\title{
Keywords : deformation / damage / failure / materials / dynamic loadings
}




\section{Introduction : problématique générale et applications}

La modélisation du comportement thermomécanique des matériaux d'ingénierie s'est longtemps développée dans le cadre principal de deux grands secteurs assez cloisonnés. D’un côté, les applications industrielles classiques se cantonnaient à l'étude des sollicitations relativement lentes, dites «quasi-statiques », appliquées à l'échelle de la minute, de la seconde éventuellement. D'un autre côté, les applications militaires (et, pour une moindre part, géophysiques et astrophysiques) ont constitué le moteur principal de l'étude des sollicitations rapides, appliquées à l'échelle de la milli- ou de la microseconde, voire moins (Figure 1). Ce cloisonnement tend actuellement à s'estomper sous l'impulsion de nouvelles problématiques, dont les applications de type sécurité représentent une fraction notable. Nous donnons ci-dessous quelques exemples de telles applications, par ordre croissant de rapidité (on remarquera que, dans bien des cas, plus un phénomène est rapide, plus son intensité est élevée) :

- chutes lors d'accidents de manutention (p. ex. des bagages ou structures aéronautiques $[1,2])$;

- $\quad$ crash aéronautique [3], automobile [4, 5], ferroviaire, ou naval ;

- impacts (accidentels d'oiseaux sur verrières d'aéronefs, de projectiles lors d'actes malveillants [6])

- $\quad$ chocs thermiques $[7,8]$.

L'étude du comportement dynamique des matériaux et des structures est donc un domaine en pleine expansion. Certaines applications industrielles exploitent d'ailleurs de plus en plus des phénomènes rapides : abattage de roches par explosif, exploitation pétrolière, usinage à grande vitesse, mise en forme (p. ex. forgeage, extrusion, moulage sous pression), 
placage ou soudure par explosif, destruction de calculs rénaux ou biliaires par impacts laser, etc.

La problématique abordée ici concerne la spécificité des mécanismes et de la mécanique de la déformation, de la dégradation et de la rupture sous sollicitations dynamiques, en vue de dimensionner des structures ou d'évaluer leurs capacités résiduelles de résistance. Le champ classique de la mécanique des solides traite des sollicitations quasistatiques, pour lesquelles les effets d'inertie sont négligeables par rapport aux autres chargements. Le dimensionnement des structures et des systèmes fait alors appel à divers outils, tels que la prévision de l'amorçage de macrofissures à l'aide de la théorie de l'endommagement [9], la tenue de structures fissurées à l'aide de la mécanique de la rupture (linéaire ou non) $[10,11]$, la théorie du flambement, des charges limites, ou encore la tenue à la fatigue. Dans ce dernier cas, qui invoque des sollicitations cycliques et/ou vibratoires, on néglige le plus souvent l'effet de la fréquence [12].

Les sollicitations rapides sont souvent qualifiées de « dynamiques », lorsque les effets d'inertie ne peuvent plus être négligés, et que l'énergie cinétique mise en jeu n'est plus négligeable vis-à-vis de l'énergie de déformation. Le dimensionnement des structures est beaucoup plus difficile à effectuer. Le champ vibratoire (étude des fréquences et des modes propres) pour lequel, le plus souvent, on néglige les régimes transitoires pour ne retenir que le régime permanent ne sera pas considéré ici.

\subsection{Quand peut-on parler de régime dynamique?}

Il apparaît dès à présent nécessaire de préciser ce que l'on entend sous le terme « dynamique ». Une modélisation raisonnable (si possible simple, nous y reviendrons) ne peut pas décrire le comportement d'un matériau ou d'une structure pour n'importe quelle 
sollicitation: il s'agit donc de bien définir, pour dimensionner une structure ou un matériau, quels sont les chargements susceptibles d'être rencontrés, non seulement dans son cadre d'emploi normal mais encore dans des situations extrêmes ou accidentelles (pour des raisons de sécurité généralement).

Si l'on raisonne en termes de comportement de la structure (qui peut éventuellement contenir des défauts), les principaux paramètres qui caractérisent une sollicitation sont, en première approche, au nombre de trois : l'amplitude du chargement $\mathrm{Q}$, sa durée $\Delta$ et la façon dont le chargement varie au cours du temps, variations décrites par un temps caractéristique T.

La réponse de la structure peut être schématisée par trois paramètres : $\lambda$ une dimension caractéristique de la structure ou d'une hétérogénéité caractéristique du matériau (celui-ci est alors considéré, à l'échelle d'observation comme une structure), $\delta$ un temps caractéristique de la structure qui correspond au temps mis par une impulsion appliquée à la structure pour parcourir une distance de l'ordre de $\lambda$ et $\mathrm{Q}_{\max }$ une amplitude de chargement admissible pour la structure au sens où l'intégrité de la structure est assurée pour une amplitude inférieure à $\mathrm{Q}_{\max }$. Cette notion de chargement admissible peut faire référence à un critère de résistance locale, d'endommagement critique [9], voire de ténacité dans le cas où des notions de mécanique de la rupture $[10,11]$ sont appliquées dans ce que l'on appelle la tolérance au dommage. Dans ce dernier cas, l'amplitude de chargement doit être nécessairement pondérée par la taille des fissures réellement préexistante ou pouvant potentiellement se manifester.

La comparaison des paramètres caractéristiques de la structure et du chargement permet de classer les différents types de chargements. Si l'intensité du chargement est faible devant $\mathrm{Q}_{\max }$, la réponse de la structure est généralement linéaire et devient non linéaire pour une amplitude de l'ordre de $\mathrm{Q}_{\max }$. Si $\Delta$ est grand devant $\mathrm{T}$, on suppose que l'intensité du 
chargement $\mathrm{Q}$ est suffisamment faible devant $\mathrm{Q}_{\max }$ pour que la durée de vie de la structure puisse effectivement être de l'ordre de $\Delta$, voire plus, et donc supérieure à la période caractéristique $\mathrm{T}$ du chargement. Si $\mathrm{T}$ est grand devant $\delta$, la variation temporelle de la sollicitation est suffisamment lente pour que la structure puisse suivre de façon globale ces variations : les phénomènes de propagation n'ont pas à être pris en compte, même si l'énergie cinétique ne peut pas forcément être négligée.

Dans le cas où T est inférieur ou de l'ordre de $\delta$, les effets de propagation doivent être pris en compte : la réponse de la structure est qualifiée de dynamique et les phénomènes de propagation d'ondes sont obligatoirement intégrés à la description de l'évolution de la structure. Lorsque l'amplitude des sollicitations est faible, la réponse de la structure est linéaire et, si le chargement est périodique, la réponse est qualifiée de vibratoire, état asymptotique faisant suite à une période transitoire.

Lorsque la sollicitation est quelconque (non périodique), le phénomène est qualifié de dynamique transitoire. Dans ce cas, le chargement peut généralement se décomposer en deux phases. Prenons par exemple le choc de deux structures; il existe une phase de mise en charge dont la durée est inférieure au temps $\delta$ caractéristique du système, suivie d'une phase de mise en équilibre durant laquelle le chargement global varie peu : le temps caractéristique associé au chargement devient dans cette phase de l'ordre de $\Delta$ et supérieur à $\delta$ (ceci n'est évidemment vrai que globalement; localement, les chargements sont extrêmement instationnaires). Cette mise en équilibre de la structure (homogénéisation des champs mécaniques) nécessite généralement quelques dizaines de $\delta$; les instants suivants peuvent alors être décrits sans tenir compte de phénomènes de propagation, dans la mesure où le chargement ne conduit pas à la ruine de la structure (celle-ci engendre souvent des ondes de relaxation). 
Cette analyse mécanique n'est cependant pas suffisante et il faut y adjoindre une analyse des phénomènes thermiques, induits par le couplage thermomécanique dans le processus de déformation. De la même manière, on peut définir un temps caractéristique de la diffusion de la chaleur sur une longueur $\lambda$. Ce temps est supposé court devant la durée d'une sollicitation quasi-statique si bien que l'on peut supposer le processus isotherme. En dynamique transitoire, c'est l'inverse et on fait généralement l'hypothèse d'adiabaticité du phénomène. Entre ces deux cas limites, ou pour des sollicitations thermiques très violentes (choc laser par exemple), toutes les situations sont possibles et une analyse thermomécanique couplée peut se révéler nécessaire. On notera que, pendant le temps caractéristique $\mathrm{T}$, des échanges de chaleur peuvent se produire à petite échelle (par exemple à l'échelle de l'hétérogénéité d'une microstructure), ce qui n'est pas forcément sans conséquence localement. En ce sens, il faut préciser à quelle échelle on exploite l'hypothèse d'adiabaticité.

Le terme «dynamique» recouvre donc des notions de mécanique, de thermodynamique, et de physique assez variées. Pour se donner une idée concrète des implications de l'analyse précédente, voici quelques exemples. Les spécialistes s'accordent à considérer comme dynamique tout processus de déformation invoquant des vitesses de chargement supérieures à $1 \mathrm{~s}^{-1}$ (ou $100 \%$ de déformation par seconde $)^{1}$. L'usage veut que l'on distingue les régimes dynamiques « lent, moyen et rapide ». Le régime dynamique lent (entre 1 et $10 \mathrm{~s}^{-1}$, par exemple, chute accidentelle d'objets) est proche du processus quasi-statique et peut s'appréhender avec les mêmes outils, et en particulier ses techniques expérimentales. Ces vitesses de sollicitation correspondent généralement aux capacités maximales des moyens d'essais (électrohydrauliques asservis) classiques et de leur instrumentation. Le régime

\footnotetext{
1 À noter qu'en toute rigueur, les effets dynamiques sont induits par l'accélération et non la vitesse de sollicitation, au moins sur le plan de la structure
} 
dynamique moyen (entre 10 et $1000 \mathrm{~s}^{-1}$ ) se caractérise par une échelle temporelle de l'ordre de la milliseconde; le phénomène de crash se situe typiquement dans cette catégorie. Le régime dynamique rapide (au-delà de $10^{4} \mathrm{~s}^{-1}$ ), à l'échelle de la microseconde, est caractérisé par le phénomène de propagation d'ondes mécaniques, et en particulier d'ondes de choc. Dans ce dernier cas, l'une des particularités des chargements, transitoires par essence, est de mettre en jeu des pressions et des taux de triaxialité très élevés. Entrent dans cette catégorie les phénomènes d'impacts balistiques ou d'explosions. L'extrémité de cette gamme $\left(10^{6}\right.$ à $\left.10^{8} \mathrm{~s}^{-1}\right)$, dont le temps caractéristique peut atteindre la nanoseconde, est souvent qualifiée d'hydrodynamique, et correspond à des phénomènes d'une extrême violence (un bon exemple est représenté par les impacts météoritiques) : les matériaux se comportent essentiellement comme des fluides, éventuellement hors d'équilibre thermodynamique, et peuvent même relever de la physique des plasmas.

\subsection{Comment modéliser « en dynamique »?}

On se cantonne dans ce qui suit aux domaines les plus classiques des chargements dynamiques moyen (crash) et rapide (impacts, explosions), et aux matériaux les plus employés (métaux, bétons, céramiques, verres, polymères et composites). On a vu plus haut que l'échelle temporelle à considérer est comprise entre la milli et la microseconde. On notera que certains phénomènes se produisant pour des temps plus longs peuvent être inhibés. Par exemple, le phénomène de propagation de fissure unique est souvent remplacé par celui de multifissuration [13] et/ou de multi-fragmentation. Le cas de l'écaillage du verre est évocateur; sous choc, il ne se dégrade pas, et les tensions dynamiques rencontrées dans le processus d'écaillage le font s'endommager par microcavitation, de manière similaire aux métaux [14]. La classique hypothèse du «maillon le plus faible », appliquée à l'échelle 
spatiale d'une structure, n'est plus valable. Pour les durées de sollicitations qui nous intéressent, les informations thermomécaniques n'ont que peu de temps pour se propager: elles n'affectent qu'un voisinage, plus ou moins immédiat, de la zone sollicitée et non plus l'ensemble de la structure. On constate que la notion d'échelle spatio-temporelle est à nouveau le point clef pour une analyse pertinente du phénomène : la seule notion d'échelle spatiale, classiquement utilisée en micromécanique, ne suffit plus. À titre d'exemple, comment choisir un volume élémentaire représentatif d'une microstructure donnée, lorsque le chargement ne s'applique que sur une fraction d'hétérogénéité, comme c'est le cas pour certaines ondes de choc (dans leur direction de propagation)?

Si l'on raisonne en terme de comportement de matériaux, on peut comparer le temps caractéristique $\mathrm{T}$ de la sollicitation avec un (éventuellement plusieurs) temps caractéristique(s) $\tau$ des processus de déformation et/ou de dégradation du matériau. Dans le cas où $\mathrm{T} \gg>\tau$, les processus peuvent se produire quasi-indépendamment de la vitesse de chargement. Dans le cas où $\mathrm{T}<<\tau$, les processus sont figés : le matériau est métastable vis-àvis de ces processus et, le plus souvent, de nouveaux processus de déformation viennent prendre le relais : la phénoménologie change, et il est peu probable que les mêmes lois de comportement restent valables. Dans les cas intermédiaires, les phénomènes dépendent fortement du temps : ils sont sensibles à la vitesse de déformation. Dans certains cas, l'état thermodynamique peut s'écarter fortement de l'équilibre, et les outils thermodynamiques usuellement employés ne s'appliquent pas forcément directement. Comme on le voit, les outils de modélisation thermomécanique doivent eux aussi faire l'objet de développements.

Dans le cas des chargements dynamiques, comme dans les autres, prévoir la tenue de structures, suppose de répondre aux interrogations suivantes (Figure 2) : 
- Quelles sont les sollicitations que doit subir la structure, quelles sont les échelles caractéristiques de la structure?

- Quel type de modèle choisir ? À quelle(s) échelle(s) doit-on étudier le comportement, la dégradation et la rupture de matériaux sous chargements dynamiques?

- Comment expérimenter, identifier et valider un modèle ?

- Comment implanter numériquement le modèle ? Quels codes de calcul utiliser et quels développements spécifiques doit-on effectuer?

Pour tenter de répondre à ces questions, il s'agit tout d'abord d'analyser les particularités concernant l'expérimentation, la modélisation et la simulation numérique relatives aux sollicitations dynamiques. Dans un second temps, on montrera les acquis et les déficits que l'on peut dégager dans l'état actuel de la prévision du comportement, de l'endommagement et de la rupture sous sollicitations dynamiques. À partir de cette analyse, des directions d'études seront ébauchées afin de combler les manques.

\section{Quelles sont les particularités liées aux sollicitations dynamiques ?}

Dans un premier temps, on notera que les stratégies expérimentales, de modélisation et numériques sont souvent spécifiques aux sollicitations dynamiques.

\subsection{L'expérimentation}

Dans le domaine des sollicitations rapides, l'expérimentation est difficile car les temps caractéristiques sont très courts, souvent onéreuse et peu fiable (nécessité de faire des essais à 
l'échelle 1 de toute la structure [4]). Ces essais se font en tout ou rien (tenue d'une caisse automobile ou ferroviaire) ou en instrumentant la structure.

On distingue assez systématiquement les vitesses de déformations inférieures à $10 \mathrm{~s}^{-1}$, pour lesquelles les machines d'essais ont une architecture assez proche de celles qui sont utilisées pour caractériser le comportement et la rupture des matériaux sous sollicitations quasi-statiques, bien que l'inertie propre de la machine d'essai rende la décharge difficile. D'autre part, pour des sollicitations supérieures à $100 \mathrm{~s}^{-1}$, on utilise généralement un montage de barres de Hopkinson-Kolsky [15, 16] qui permet, selon le dispositif, d'appliquer un chargement de compression, traction ou torsion. Pour le montage de compression, un échantillon du matériau à caractériser est placé entre deux barres et une troisième barre, coaxiale, vient impacter cet ensemble (Figure 3) ; un temps caractéristique de la sollicitation est, par exemple, la durée du front de montée de celle-ci (quelques $10 \mu \mathrm{s}$ ) ou sa durée (de l'ordre de $100 \mu \mathrm{s}$ ) qui est inférieure au temps d'aller-retour d'un signal dans une barre (de l'ordre de la milliseconde). À la différence d'un moyen d'essai classique, on doit donc prendre explicitement en compte la propagation des ondes dans l'ensemble du dispositif, ce qui justifie sa géométrie très simple. L'analyse de ces ondes ainsi que le fonctionnement et les limites du dispositif sont décrits précisément dans [17]. En ce qui concerne l'échantillon, on peut, en revanche, dans bien des cas supposer que son évolution se fait de manière homogène (mais à vitesse de déformation élevée) sauf peut être en début de chargement puisque le temps caractéristique pour un échantillon métallique est généralement de l'ordre de la microseconde, c'est-à-dire faible devant les temps caractéristiques de la sollicitation. Le dépouillement de ces essais devient en revanche bien plus délicat lorsque l'échantillon n'est pas en équilibre quasistatique : il faut le traiter comme une structure (sollicitée en dynamique) et non plus comme 
un élément de matière [18] et une approche inverse doit être utilisée pour identifier les paramètres du comportement [19].

Au-delà de $1000 \mathrm{~s}^{-1}$, l'un des moyens privilégiés d'investigation est le chargement par onde de choc, obtenu soit par impact de plaques à l'aide de lanceurs à poudre ou à gaz, soit par explosif [20]. Si le choc plan, régime de sollicitation en déformation uniaxiale, reste l'essai le plus pratiqué, des techniques proches, telles que l'impact incliné ou la propagation d'ondes sphériques divergentes [21], permettent d'étendre quelque peu la gamme des trajets de chargement. Ces essais ne sont pas homogènes, et s'exploitent et s'instrumentent de façon très spécifique. Le cas particulier des ondes de choc stationnaires permet de reconstruire, par la technique d'analyse lagrangienne [22] et à l'aide d'une métrologie appropriée, les trajets contrainte-déformation.

Dans tous les cas, conduire des essais interrompus (par exemple pour faire des décharges) est délicat mais possible dans certains cas (e.g., impact de plaques). Les essais multiaxiaux sont actuellement réservés aux sollicitations quasi-statiques avec des machines électrohydrauliques asservies [23]. On notera également que contrairement aux essais quasistatiques où les variables de contrôle durant l'essai sont assez bien identifiées et maîtrisées (déplacement, déformation ou effort), ceci est loin d'être le cas des essais dynamiques pour lesquels par exemple le contact entre surfaces permettant l'application du chargement ne reste pas forcément maintenu durant tout l'essai.

Les mesures pour tous ces essais sont délicates car même la réponse d'un capteur piézo-électrique, supposée linéaire dans le cas quasi-statique, peut devenir non-linéaire du fait des phénomènes d'inertie ou du comportement proprement dit des matériaux constitutifs [24, 7]. D'autre part, les mesures locales ne sont possibles que dans quelques cas très particuliers. On utilise souvent des informations globales en face arrière comme des vitesses particulaires. 
On peut aussi, dans certains cas, noyer des jauges de contraintes dans le matériau [25, 26] sans toujours bien connaître leur influence sur leur voisinage. Ce point peut être vu sous l'angle général de la perturbation du milieu par la mesure, ce qui est également le cas en sollicitation quasi-statique si l'on cherche des mesures in situ. Pour le choc plan, voire sphérique, certaines techniques, telles que les jauges piézorésistives pour la mesure de contraintes, ou les jauges électromagnétiques pour la mesure de vitesse matérielle, donnent des résultats tout à fait satisfaisants, et des précisions de mesure acceptables, en général inférieures à 5\% lorsqu'elles sont bien maîtrisées. Leur implantation dans des matériaux très hétérogènes, tels que le béton, sort évidemment de ce cadre, puisque la dimension de l'hétérogénéité (granulat) est alors bien supérieure à celle de la jauge.

Les mesures de température sont longtemps restées hors de portée. Toutefois, le développement de techniques optiques $[27,28]$ devrait permettre de combler le manque, en réalisant des mesures de surface ou d'interface, suffisamment précises et très bien résolues en temps. Il est toutefois nécessaire d'établir la relation entre température de surface et température interne au matériau. Le développement de techniques expérimentales nouvelles, résolues en temps aussi bien qu'en espace, et fiables, apparaît (aujourd'hui plus que jamais) indispensable, surtout si l'on considère que les instruments à mettre en œuvre varient avec les échelles temporelles caractéristiques, et que les couplages thermomécaniques ajoutent souvent une variable au problème.

Si les moyens d'étude et de caractérisation paraissent assez restreints, il existe en revanche de nombreuses techniques permettant de réaliser des essais dits globaux bien instrumentés : essais de pénétration/perforation [29], impacts sur la tranche [30], essais de Taylor [31], essai de compression électromagnétique [32], etc. Ces techniques cherchent en général à reproduire, en les simplifiant et en permettant la mesure, des chargements réels. Les 
métrologies associées sont souvent optiques, donc sans contact : moiré [33], interférométrie Doppler-laser [34], cinématographie ultra-rapide, radiographie-éclair [20]. Elles donnent accès à des grandeurs globales, et non plus locales (champ de fissuration surfacique, vitesse de surface libre ou d'interface, vitesse de pénétration, vitesse résiduelle de fragments, etc.). On se trouve là dans une démarche de validation, où la confrontation expérience-calcul tient une place éminente, ce qui montre bien le rôle du calcul en dynamique. Moyennant une approche inverse, certaines expériences permettent une identification partielle, bien que la prudence soit de rigueur pour de telles approches (voir par exemple le cas de l'essai de Taylor [35]). Enfin, les analyses post-mortem peuvent s'avérer délicates dans le cas dynamique. En effet, la récupération de l'éprouvette ou de la structure sollicitée n'est pas toujours aisée et nécessite des précautions importantes pour éviter les artefacts (voir par exemple [21, 36]). Ceci augmente d'autant les coûts mis en jeu lors d'un essai dynamique, qui sont de toute façon élevés, étant donné qu'aux grandes vitesses, une grande partie des montages expérimentaux est détruite en même temps que l'échantillon testé.

\subsection{Comportement et ruine}

Le comportement des matériaux peut être différent dans le cas de sollicitations quasistatiques et dynamiques. A titre d'illustration, on notera que les matériaux habituellement réputés fragiles ne subissent plus de rupture brutale, mais plutôt de la multifissuration, et relèvent donc d'un comportement quasi-fragile (voir le cas du carbure de silicium, Figure 4 [13]), voire même ductile (voir le cas du verre [14]).

L'adiabaticité des phénomènes rapides rend indispensable la prise en compte de la dépendance du comportement par rapport à la température et de la dissipation mécanique dans l'équation de la chaleur. La situation se complique encore dans le domaine dynamique moyen, 
où il devient nécessaire de prendre en compte les échanges de chaleur dans une analyse thermomécanique couplée (situation intermédiaire entre trajets isotherme et adiabatique). Ceci rend, dans certains cas, l'exploitation de résultats expérimentaux particulièrement malaisée, à cause de l'inhomogénéité des champs thermomécaniques. La prise en compte des transferts de chaleur doit également intervenir en dynamique rapide, lorsque les phénomènes en jeu se situent à très petite échelle (de temps et d'espace).

Certains phénomènes typiques de chargements rapides se produisent sous forme localisée. C'est le cas du «cisaillement adiabatique » (Figure 5), génération de bandes de cisaillement ultra fines, prélude à des ruptures catastrophiques, provoquées par l'adoucissement thermique associé à l'échauffement adiabatique par dissipation [37]. C'est également le cas du mécanisme d'écaillage (Figure 6). Des zones de forte traction triaxiale très localisées se développent par croisement d'ondes de détente consécutives à des impacts ou des explosions. Le phénomène, très rapide, n'autorise pas de fissuration macroscopique, trop lente, et on assiste à la germination, croissance et coalescence de microcavités ou de microfissures. À noter par ailleurs que tel matériau, réputé ductile en quasi-statique, peut relever d'un endommagement quasi-fragile par microfissuration en dynamique [38]. On retrouve cette situation sur le polycarbonate soumis à un impact: le mécanisme de dégradation fait apparaître, lorsque la vitesse du projectile est suffisante, un réseau de microfissures absent aux faibles vitesses.

\subsection{Modélisation}

Actuellement plusieurs pistes sont suivies pour appréhender les problèmes de comportement et de rupture sous sollicitations dynamiques. Les approches continues dominent comme pour la résolution des problèmes quasi-statiques. Cependant on notera aussi 
l'émergence d'approches discrètes. D'autre part, la prévision de la rupture à partir d'approches continues pose le même problème qu'en sollicitation quasi-statique : comment passer d'une description continue tridimensionnelle à l'apparition d'un objet bidimensionnel (i.e., une fissure, une bande de cisaillement) ? Ce dernier problème, difficile et qui considère forcément une fissure préexistante, n'a trouvé de réponse satisfaisante pour des sollicitations dynamiques que dans le cadre de la mécanique linéaire de la rupture fragile [39] ou lorsque la zone élastoplastique reste confinée au voisinage de la pointe de fissure [40]. Il est intéressant de noter que cette approche repose sur une analyse à deux échelles spatio-temporelles : le voisinage élastoplastique du fond de fissure est traité en quasi-statique tandis que le reste de la structure se trouve en régime élastodynamique.

\subsection{Simulation}

Comme pour la modélisation, plusieurs directions sont actuellement envisagées [41]. La formulation du problème de dynamique transitoire conduit à un système d'équations aux dérivées partielles non linéaires. Dans les codes de calcul, sa résolution systématique passe obligatoirement par sa discrétisation. Cette discrétisation est nécessaire non seulement en espace mais aussi en temps. Dans ces codes de calcul dits « continus » où la discrétisation en espace repose dans la plupart des cas sur la méthode des éléments finis (MEF) [42] tandis que la discrétisation en temps (schéma d'intégration de type Newmark [43]) est faite de manière découplée, par différences finies (une discrétisation espace-temps simultanée est possible mais rarement employée). Ces codes sont qualifiés de « continus » puisqu'ils imposent a priori (c'est-à-dire hormis algorithme spécifique) la conservation de la connectivité initiale du maillage (donc généralement de la matière, cf. plus bas). Les discrétisations en espace (caractérisée par la «taille» de maille) et en temps (caractérisée par le pas de temps) vont 
conditionner conjointement l'algorithme de résolution. La stabilité de celui-ci est une condition nécessaire : cette condition asymptotique, qui stipule que la solution numérique ne doit pas diverger dans le temps, peut être inconditionnelle ou conditionnelle sur le pas de temps, pas de temps dont dépend directement le coût du calcul. Dans ce dernier cas, le pas de temps ne doit pas dépasser une borne, fonction des (deux) paramètres définissant le schéma aux différences finies en temps et des propriétés spectrales du modèle EF (période du mode propre le plus élevé [44]). Le schéma d'intégration en temps est dit explicite lorsque l'état au pas de temps $n+1$ est obtenu par simple substitution, dans les équations, de l'état au temps $n$, opération immédiate lorsque la matrice de masse est concentrée (la masse est concentrée aux nœuds, c'est-à-dire sur les termes diagonaux de la matrice ; la matrice d'amortissement étant généralement diagonale aussi, leurs inversions sont triviales). Le schéma est implicite dans les autres cas ; la nécessité d'inverser certaines matrices rend les calculs plus coûteux. Pour les schémas explicites, conditionnellement stables, l'enveloppe de la condition de stabilité s'exprime par la condition de Courant, Friedrich et Levy (CFL) [45] qui stipule que, sur la durée d'un pas de temps, un front d'onde élastique ne doit pas parcourir une distance supérieure à la maille EF. Cela ne suffit pourtant pas et il faut, pour évaluer la qualité de la simulation numérique, en contrôler la précision, c'est à dire l'écart entre la solution exacte et la solution discrète. Il faut pour cela assurer la consistance du schéma numérique, propriété locale qui mesure si la solution exacte continue vérifie, lorsque le pas de temps tend vers zéro, le schéma discret. L'erreur commise en injectant la solution exacte dans le schéma discret est appelée erreur de troncature. Le cumul de ces erreurs permet, en supposant le schéma stable, d'en évaluer la précision. La stabilité et la consistance du schéma impliquent sa convergence. Se donner un critère de précision a pour conséquence de borner le pas de temps : le majorant dépend alors de la précision à atteindre mais aussi de la plus petite période caractéristique de 
la sollicitation (temps $\mathrm{T}$ défini dans la première partie) : en conséquence, il y a compétition entre les conditions de stabilité et de précision. Le coût intrinsèque d'un pas de temps dans un schéma implicite étant, comme on l'a dit, bien plus élevé qu'en explicite (de l'ordre du carré de la largeur de bande du système à inverser) et que l'on cherche généralement à simuler un phénomène sur une durée donnée, la méthode implicite devient compétitive lorsque la sollicitation n'est pas trop «brutale». Tous ces résultats sont établis pour des systèmes linéaires et les non linéarités fortes rencontrées dans la pratique imposent de les prendre plutôt comme des tendances en prenant une marge de sécurité sur le pas de temps maximal.

Selon le choix des paramètres le caractérisant, le schéma d'intégration en temps permet de faire apparaître une viscosité numérique. Celle-ci agit comme un filtre passe-bas qui atténue et permet le cas échéant de contrôler certaines oscillations (bruit) de la solution numérique. Pour la discrétisation spatiale, il existe deux grands types d'approches: l'approche dite lagrangienne, où le maillage est lié à la matière, et l'approche eulérienne, où la matière se déplace au travers d'un maillage fixe. Cette distinction correspond à des points de vue plutôt de mécanique du solide (lagrangienne) ou de mécanique des fluides (eulérienne). Lorsque les déformations sont importantes, l'approche lagrangienne (dite « réactualisée », puisque le problème mécanique est écrit sur la configuration « actuelle », par opposition à une formulation lagrangienne «totale » qui fait, à chaque instant, référence à la configuration initiale) impose au maillage de fortes distorsions qui ont pour effet d'altérer le conditionnement et la précision du schéma numérique. La solution est donc de modifier le maillage, périodiquement ou à chaque pas de temps. On peut bien entendu ajouter des éléments dans les zones très déformées mais les changements de topologie occasionnés (connectivité et types d'éléments finis) et la nécessité de réaliser cette opération de façon automatique rendent cette tâche délicate. L'alternative consiste à conserver la topologie du 
maillage initial mais à ne pas l'astreindre à la cinématique matérielle en évitant ainsi de trop fortes distorsions : ce cas de figure est celui des méthodes ALE (Arbitrary Lagrangian Eulerian) qui constituent un intermédiaire entre les formulations lagrangienne et eulérienne [46]. Dans les deux cas de figure (ainsi que pour la formulation purement eulérienne), se pose le problème de la projection des variables mécaniques d'un maillage sur l'autre, lié au fait qu'il existe un flux de matière à travers les éléments. Ce problème devient particulièrement crucial pour les variables non conservatives (variables internes par exemple) et est la source de diffusion numérique qui occasionne une perte de précision.

Les codes « continus » restent pourtant les outils les plus utilisés. Ils sont en effet capables de traiter des problèmes avec de très nombreux degrés de liberté (jusqu'à plusieurs dizaines de millions). À ce titre, la rapidité de calcul est primordiale, et on cherche généralement à restreindre le nombre de points d'intégration (en particulier pour les schémas explicites qui réalisent un nombre élevé d'itérations). La contrepartie de ce gain en temps, est l'apparition de modes de déformation parasites, tels que le mode dit de « hourglass », qui permet à une maille rectangulaire à l'origine de prendre une forme de sablier, pour prendre un exemple bidimensionnel. Ces modes parasites, d'origine purement numérique, peuvent dans certains cas prendre une ampleur telle qu'ils s'avèrent totalement rédhibitoires pour la représentativité des calculs [5]. Ils imposent l'usage d'algorithmes de contrôle spécifiques (efforts de rappel qui limitent l'amplitude des modes dits de «hourglass », projection orthogonale à ces modes, par exemple).

Un autre aspect que l'on peut mentionner et qui conditionne la qualité de la simulation numérique est celui de l'algorithme qui décrit le comportement du matériau. Ce point n'est pas spécifique des codes de dynamique mais le nombre important d'itérations en temps peut, toujours pour des raisons de coût, pousser à une simplification de l'intégration de l'équation de 
comportement. L'exemple typique (quasi-statique) est celui de la plasticité où l'étape de projection sur le convexe de plasticité peut être implicite (et dans ce cas inconditionnellement stable) ou non. En dynamique, la prise en compte de l'effet d'adoucissement thermique ou de la dépendance en vitesse de déformation dans une loi de type Johnson et Cook [47] peut s'écrire de façon implicite ou explicite, avec un coût de calcul moindre dans le second cas.

Comme on l'a déjà mentionné, les codes continus conservent la topologie de maillage initiale : la limitation majeure qui en découle réside dans l'impossibilité de créer de façon simple et automatique des zones de discontinuité. Pour une discrétisation eulérienne, cette difficulté rejoint celle de la gestion des interfaces entre deux (plusieurs) matériaux au travers d'un même élément. Dans une approche lagrangienne, des éléments finis « enrichis » peuvent pallier ce problème, on peut imaginer un remaillage local ou encore un processus d'érosion dans lequel on supprime des éléments; dans tous les cas, la difficulté qui en résulte se retrouve dans la gestion des nouvelles surfaces libres créées. La gestion du contact (et par conséquent de l'auto-contact) entre ces surfaces est relativement délicate en dynamique; le contact de deux solides engendre, en effet, une sollicitation dont le spectre contient des fréquences (arbitrairement) élevées, ce qui a pour effet immédiat de mal conditionner le pas de temps vis à vis de la précision du schéma, comme nous l'avons vu plus haut (voir [48]).

Les codes discrets reposent, eux, sur la gestion d'interfaces de contact. Ils consistent à modéliser toutes les fissures en les laissant apparaître aux frontières d'un maillage classique. La génération de la fissuration est donnée par les lois d'interfaces (très simples actuellement). On notera que le temps de calcul est principalement conditionné par les contacts aux interfaces, dont la gestion devient alors particulièrement cruciale [49].

Cette liste non exhaustive [41] montre que le paysage des simulations numériques en dynamique est loin de faire l'unanimité dans la communauté scientifique. L'existence de 
différentes approches montre que la prévision de la dégradation et de la rupture des matériaux n'est pas résolue et n'a certainement pas atteint un degré de convergence suffisant pour qu'une approche cohérente soit suivie d'un bout à l'autre lors d'une simulation.

Cet exposé formel de chaque composante nécessaire au développement d'approches prédictives de la tenue de structures soumises à de sollicitations dynamiques ne saurait ignorer les interactions possibles et souhaitables entre elles (Figure 7). Ceci d'autant plus que, dans le domaine dynamique, l'expérience est davantage tournée vers la validation que vers l'identification.

\subsection{Stratégies de modélisation}

Tout modèle nécessite l'identification de paramètres passant par de l'expérimentation et de la mesure directe ou indirecte (point 1 de la Figure 7). Cette identification peut aussi s'accompagner d'une étude de sensibilité des différents paramètres. Faite trop rarement, elle permet de dégager les paramètres influents des paramètres secondaires (hiérarchisation des paramètres). Cette analyse peut aussi permettre de simplifier un modèle existant ou en cours d'écriture. Ce souci de simplification constitue à l'heure actuelle une demande forte de la part des utilisateurs, qui n'est pas simple à satisfaire dans le cas de modèles dynamiques souvent caractérisés par beaucoup d'empirisme et peu de physique. D'autre part, un type de modélisation ou un mécanisme de dégradation choisi peut donner lieu à une expérimentation spécifique (cf. Figure 4 dans le cas de céramiques et de verres).

Une fois le modèle développé, il est implanté dans un code de calcul (point 2 de la Figure 7). Son implémentation est plus ou moins facile selon le code de calcul choisi et l'architecture correspondante. Certains codes autorisent la programmation de lois de 
comportement par des personnes extérieures; d'autres nécessitent de faire appel aux développeurs du code. La validation, par comparaison expérience-calcul, intervient in fine.

Une simulation numérique peut également être utilisée dans le cas d'identification inverse des paramètres d'un modèle à l'aide d'essais non homogènes (point 3 de la Figure 7). Ceci peut être le cas dans des situations apparemment classiques (e.g., essais aux barres de Hopkinson-Kolsky $[18,19])$. Inversement, la conception de nouveaux essais passe souvent par des simulations de prédimensionnement tant à l'aide de calculs élastiques linéaires bidimensionnels ou tridimensionnels que non-linéaires dans certains cas.

On notera toutefois que l'architecture de nombreux codes de dynamique ne permet pas l'implantation de certaines formes de lois de comportement ou, au moins, leur impose des limitations. Le choix d'un type de code de calcul peut également conditionner le type de modélisation (par exemple discrète ou continue). La stratégie de modélisation est dans ce cas subordonnée à la disponibilité du code. Ce point mérite d'être mentionné, car la logique voudrait plutôt que l'on choisisse le code en fonction de l'application envisagée. Les investissements relatifs à l'acquisition du logiciel, sa prise en main, son utilisation et sa maintenance sont toutefois tels que, dans la plupart des cas, un compromis doit être envisagé.

\section{Quels acquis, quels manques pour quels matériaux ?}

Plusieurs stratégies sont possibles pour traiter le comportement, la dégradation et la rupture sous sollicitations dynamiques. On oppose souvent les approches dites phénoménologique et physique. La modélisation phénoménologique se base sur un ensemble de résultats expérimentaux macroscopiques dont elle réalise l'interpolation; elle intègre le plus souvent une connaissance minimale et implicite des mécanismes microscopiques les plus saillants sans entrer dans le détail, mais lui conférant a priori des capacités d'extrapolation 
raisonnables. La modélisation physique cherche à prendre en compte les mécanismes physiques microscopiques se produisant dans le matériau, la classe de matériaux considérée, ou la structure analysée. Ses capacités d'extrapolation devraient théoriquement être meilleures que celles de l'approche phénoménologique. Toutefois, étant données les limitations des techniques expérimentales d'investigations et des formulations micromécaniques afférentes, leur formulation tridimensionnelle nécessite des hypothèses relativement fortes.

Ces choix se posent à chaque étape de la démarche précédente (points d'interrogation de la Figure 2):

- Expérimentation : les mesures sont souvent ponctuelles et globales (déplacement ou vitesse) et peu nombreuses car difficiles et coûteuses. Dans certains cas, des mesures de champ plus complètes peuvent être conduites (Figure 8);

- Modélisation : elle peut être soit de type global et analytique dans une démarche de pré-dimensionnement [50], soit de type local et phénoménologique (voir par exemple les lois de comportement de métaux de Johnson et Cook [47] ou d'endommagement du verre [51]) ou basée sur la modélisation des phénomènes physiques quasi-fragiles [6] ou ductiles (modèle de Gurson [52] appliqué aux alliages métalliques [50, 23]) ;

- Simulation : il existe de la part de certains utilisateurs une demande de prise en compte des contraintes résiduelles imposées (béton précontraint [53]) ou subies lors du processus de fabrication (p. ex. procédé de rivetage de panneaux dans le domaine aéronautique [3]). Cette prise en compte peut avoir des conséquences sur le choix du modèle de comportement (e.g., matériaux choqués c'est-à-dire modifiés par le passage d'une onde de choc). Ainsi des modèles d'écrouissage isotropes sont à la base de très nombreux modèles élasto(visco)plastiques lorsque l'état initial du matériau n'est pas 
pris en compte. Par contre, ces modèles deviennent insuffisants lorsque l'étape d'élaboration est considérée (on peut faire appel à des modèles d'écrouissage cinématique et/ou anisotropes).

De nombreuses questions restent ouvertes dans ce domaine. Dans ce qui suit, nous distinguerons quatre classes de matériaux pour lesquelles les avancées actuelles sont très variables, et peuvent conduire à des stratégies d'étude très différentes.

\subsection{Les métaux}

Pour les métaux, on distinguera trois types de problématiques pour lesquelles les acquis ont atteint différents degrés de maturité. En ce qui concerne le comportement, les phénomènes non-linéaires liés à la plasticité des métaux sont assez bien appréhendés d'un point de vue phénoménologique et macroscopique, surtout dans la mesure où l'on se limite dans la plupart des cas à des chargements non cycliques. Dans le cas des aciers, il est nécessaire de considérer des modèles sensibles à la vitesse de déformation (e.g., modèle de Johnson et Cook [47] couramment utilisé dans les simulations de crash). Les alliages d'aluminium sont peu sensibles à la vitesse de sollicitation lorsque le comportement seul est analysé [50] bien qu'une modélisation à l'aide du modèle de Johnson et Cook [47] soit envisagée en vue de prendre en compte les effets de la température sur le comportement [54]. La modélisation de l'endommagement ductile (i.e., cavitation) est généralement de type macroscopique [55]. Ces modèles peuvent être couplés ou non aux lois de comportement nonlinéaires discutées précédemment. Le couplage comportement-ruine n'est pas toujours aisé ou possible. Par contre, la prise en compte de la microstructure, actuellement possible dans le cas quasi-statique, ou de l'état initial du matériau n'est pas encore traitée (p. ex., les alliages de titane à microstructure très variées répondent de manière complètement différente au 
cisaillement adiabatique, $([56,6])$. D'autre part, les propriétés de ténacité dynamique de matériaux (très) ductiles restent encore actuellement à l'ordre du jour [40]. Un traitement par les outils classiques de la mécanique de la rupture est illusoire et requiert des approches faisant appel à la mécanique des milieux continus (endommagement [9] ou approche locale de la rupture [57]). Les modèles de type $\operatorname{GTN}[52,58]$ reposent, en statique, sur une analyse des micromécanismes de croissance de cavités qui provoquent l'endommagement et la ruine du matériau. En quasi-statique, la contrainte est uniforme (sur l'élément de volume considéré) et une population homogène de défauts provoquera la germination simultanée de cavités sur ces défauts. En dynamique, la contrainte n'est plus uniforme et la germination des certaines cavités peut occulter, en les relaxant, certains sites d'amorçage. La croissance des cavités est influencée par la triaxialité des contraintes et les effets de (micro)inertie (c'est-à-dire à l'échelle de la cavité) font que l'extrapolation de ces modèles du quasi-statique au dynamique est encore en développement $[59,60]$.

\subsection{Les bétons, céramiques et verres}

Ces matériaux, à comportement quasi-fragile, sont aussi soumis en service à des sollicitations dynamiques. La modélisation du comportement et de la ruine a atteint un stade de maturité moindre que celui des matériaux métalliques. Des lois de comportement de type élastoplastique sont employées comme le classique "cap-model" [61] de la mécanique des sols (voir aussi [62]). L'avantage de ce type de modèle est sa disponibilité dans la plupart des codes de dynamique. Le couplage entre plusieurs mécanismes de dégradation simultanés reste délicat voire impossible. La modélisation de l'endommagement quasi-fragile (i.e., multifissuration) est généralement de type macroscopique et isotrope que ce soit pour les verres [51], les bétons [26, 53] ou les céramiques [63]. Le caractère anisotrope de 
l'endommagement quasi-fragile est encore peu décrit [6]. D’une manière plus générale, d'ailleurs, l'anisotropie de comportement n'est que très rarement traitée en dynamique. Les aspects liés à la microstructure ne sont pas très souvent pris en compte. Ceci peut devenir important dans l'optique d'une optimisation du matériau vis-à-vis d'une classe d'applications. Enfin, ni la prise en compte de divers pré-chargements (dynamiques ou non), ni la prévision des propriétés résiduelles (d'un ouvrage d'art par exemple) après sollicitation n'est faite. D'autre part, le caractère fragile induit des réponses aléatoires en termes de niveaux de rupture. Cet aspect est très rarement décrit et pris en compte ; bien que divers travaux existent sur ce thème $([64-66,13])$, ils restent relativement marginaux jusqu'à présent.

\subsection{Les polymères}

La modélisation du comportement des polymères est globalement moins avancée que celle des matériaux cristallins, même dans le domaine quasi-statique. Ces matériaux ont en effet un comportement très complexe, et sont sensibles à de nombreux paramètres, tels que la température, la vitesse de sollicitation, l'état des contraintes (et en particulier la pression). En outre, ils sont sujets au vieillissement physique et/ou physico-chimique, et sont sensibles à l'humidité. Étant donné qu'ils sont souvent hors d'équilibre thermodynamique, les outils de modélisation adéquats sont moins solidement établis que pour les matériaux cristallins. De ce fait, les modèles foisonnent, mais aucun consensus ne se dessine nettement à l'heure actuelle, même si des efforts considérables ont été consentis depuis plusieurs années, notamment par les communautés américaine et allemande.

Les polymères souples (amorphes au-dessus de leur transition vitreuse et élastomères) relèvent d'un comportement (thermo)viscoélastique non linéaire. Ils possèdent une gamme extrêmement large de temps de relaxation caractéristiques, qui dépendent eux-mêmes de 
nombreux paramètres, température et pression notamment. Même si quelques outils permettent d'en simplifier la description (en particulier le principe d'équivalence tempstempérature-pression valable pour d'assez nombreux matériaux), leur modélisation reste complexe, invoque de nombreux paramètres d'identification coûteuse, et leur simulation numérique est très consommatrice de place mémoire et de temps de calcul. De gros progrès ont été accomplis ces dernières années en direction d'une certaine unification [67,68], qui reste malgré tout lointaine. On notera que la largeur de la gamme de temps de relaxation permet l'emploi de tels modèles dans le domaine dynamique sans adaptation particulière, à condition de les identifier correctement, et de prendre en compte les couplages thermomécaniques.

Les polymères rigides (au-dessous de la transition vitreuse pour les polymères amorphes, semi-cristallins) ont un comportement un peu moins complexe qui peut être appréhendé par des modèles (thermo)élasto-viscoplastiques empiriques. Ces modèles, relativement simples, peuvent convenir à des applications d'ingénierie, à condition de les considérer comme une première approximation, et de ne pas les employer en dehors de leur domaine d'identification. Des modèles à bases physiques existent [69], et ont été employés avec succès dans le domaine dynamique [70]. Ils sont plus complexes que les précédents, et ne conviennent pas à une utilisation industrielle, sauf cas particuliers; ils sont toutefois beaucoup plus fiables et plus extrapolables.

La modélisation de l'endommagement et de la rupture des polymères est un thème relativement peu avancé, étant donnée la diversité phénoménologique constatée pour ces phénomènes. De ce point de vue, les matériaux les mieux décrits sont peut-être les élastomères chargés, dont la sécurité d'emploi a imposé l'étude intensive [71, 29, 72]. Les modèles correspondants sont généralement viscoélastiques endommageables ; très complexes, 
ils ne sont employés que pour des applications spécifiques, en quasi-statique aussi bien qu'en dynamique.

Les matériaux amortissants (élastomères, mousses), bien que d'un emploi très courant (dans l'industrie automobile par exemple), ne sont qu'assez peu étudiés hors des domaines précités, dans la mesure où leur contribution mécanique (raideur principalement) est négligeable. La structure alvéolaire ou poreuse des mousses (Figure 9) induit une échelle caractéristique qui, selon la vitesse de sollicitation, permet de les considérer comme homogènes, ou impose au contraire de les analyser comme des structures hétérogènes [73].

\subsection{Les matériaux composites et assemblages}

Enfin, nous analysons la classe des matériaux composites. La modélisation du comportement mécanique particulier lié à l'architecture de ces matériaux n'est que très peu abordée $[74,1]$. En effet, la présence simultanée de plusieurs échelles géométriques liées à la microstructure particulière (matériaux stratifiés, tissés, etc.) rend l'étude de ces matériaux particulièrement difficile, pour les mêmes raisons que celles déjà évoquées plus haut pour les mousses. Enfin les modèles (anisotropes) de rupture ne sont que peu étudiés actuellement et les propriétés résiduelles (cf. analyse pour les matériaux fragiles) après sollicitations dynamiques sont à explorer de manière plus marquée dans le futur (dans le domaine spatial notamment).

L'étude d'un matériau seul n'est pas suffisante. En effet, de nombreuses applications utilisent plusieurs matériaux :

- assemblages de matériaux (points ou cordons de soudure [4], rivetage [3], collage [4]) ; 
- concepts bicouches de blindages (association de céramiques et d'aciers à blindage, de céramiques et de composites, [6]) ou verrière d'engins civils ou militaires (plusieurs couches de matériaux transparents [3]).

Comme pour les matériaux composites, les interactions entre les différents matériaux soumis à des sollicitations dynamiques doivent être prises en compte et maîtrisées si l'on veut garantir la tenue d'une structure complexe. L'approche expérimentale repose sur les mêmes moyens que ceux utilisés pour caractériser les matériaux, hormis leurs dimensions souvent plus importantes pour solliciter une structure (e.g., puits de chute, arbalète, barres de Hopkinson de grand diamètre, machine électrohydraulique rapide). La réponse de la structure étant généralement inhomogène, les mesures (souvent globales) sont plus délicates à interpréter et le recours à la simulation numérique devient nécessaire, d'autant plus que les considérations économiques font souvent hésiter à instrumenter localement. Cet aspect numérique de l'interaction entre matériaux fait apparaître certaines difficultés, notamment liées aux contacts ou à la gestion simultanée par un code unique de plusieurs lois de comportement très différentes sur un plan algorithmique.

\subsection{Bilan}

Pour toutes les classes de matériaux, on constate un déficit de transitions d'échelle(s) en vue de la prise en compte des mécanismes microscopiques de déformation et/ou de détérioration et, plus généralement, de l'influence de la microstructure sur le comportement macroscopique des matériaux. Une étude à différentes échelles permettra un enrichissement des modèles en vue, premièrement, d'en cerner les limites et le domaine de validité et deuxièmement de savoir comment étendre ce domaine de validité. D'autre part, ceci constituerait une direction possible en vue de valider des modèles heuristiques. Par ailleurs, 
les outils mathématiques de transition d'échelle (homogénéisation) ne sont pas valables en présence d'effets d'inertie, et des développements théoriques, bien que commençant à apparaître [75], restent largement insuffisants (voir l'exemple de l'écaillage ductile [60]).

On constate deux tendances nettement marquées dans la demande. D'un côté, le besoin de lois de comportement simples, fiables et d'identification aisée se fait sentir avec beaucoup d'acuité. De l'autre, c'est l'aspect physique qui est le plus souvent souligné, avec l'espoir que la prise en compte fine d'une physique réelle conférera aux lois de comportement un caractère extrapolable. Il est a priori plus satisfaisant de procéder à la simplification $a$ posteriori d'une loi à bases physiques, que de proposer des lois phénoménologiques très probablement simplistes, et dont l'extrapolation est nettement sujette à caution. Une telle démarche est toutefois coûteuse et longue, et ne peut pas être mise en œuvre systématiquement.

\section{Quelles directions envisager?}

Pour traiter de la dégradation et de la rupture sous sollicitations dynamiques, quelques directions de réflexions peuvent être dégagées dans l'état actuel des connaissances :

- plusieurs approches de modélisation sont développées actuellement : continues (dans le cadre de la mécanique et de la thermodynamique des milieux continus), discrètes (e.g., mécanique statistique, dynamique moléculaire, modèles de fragmentation). Ces deux approches sont souvent mises en opposition. Leur domaine respectif d'utilisation n'est pas encore clairement défini et constitue un des défis futurs, surtout si l'on s'intéresse à l'obtention et au développement des modes localisés (e.g., écaillage, perforation, fissuration), précurseurs de la ruine des structures. D'autre part, sous-jacente est également l'interrogation sur le fait d'utiliser des approches probabilistes ou déterministes 
pour aborder la modélisation à base physique ;

- l'implantation dans un code de calcul n'est pas encore chose très aisée en ce qui concerne les calculs dynamiques. L'expertise acquise dans le domaine quasi-statique n'est pas toujours transposable au cas dynamique [41]. C'est en ayant ces difficultés à l'esprit que le développement de modèles de comportement et de rupture sera effectué. Même si des algorithmes fiables et précis existent, les utilisateurs cherchent le plus souvent un compromis coût-efficacité maximum, ce qui se traduit parfois par un manque de fiabilité, de robustesse, ou de confiance dans les résultats numériques.

- les changements d'échelles, notion maintenant acquise en mécanique non-linéaire des matériaux sous sollicitations quasi-statiques, trouvent ici un terrain d'application (peu exploré) plus complexe encore avec le couplage des échelles d'espace et de temps. La prise en compte de la microstructure impose une réflexion dans ce sens de même que la modélisation des dégradations qui sont souvent causées par des hétérogénéités microscopiques et qui conduisent, in fine, à la rupture macroscopique du matériau. Ce type de démarche permet d'entrevoir une clé à la construction de modèles simples voire simplifiés mais à base physique. Cependant ceci nécessitera des travaux sur le long terme. La notion de matériaux et de problèmes génériques devrait également pouvoir émerger et ainsi diminuer le nombre de paramètres à identifier pour un nouveau matériau pour lequel les utilisateurs potentiellement intéressés exigeront la loi de comportement (identifiée) avant de les proposer pour de nouvelles applications [4]. 


\section{Conclusions et perspectives : MECADYMAT un groupe de}

\section{travail dans l'AFM}

La modélisation du comportement et de la rupture sous sollicitations dynamiques (i.e., pour des vitesses de déformations supérieures à $1 \mathrm{~s}^{-1}$ ) fait le plus souvent appel à des modèles macroscopiques (et heuristiques) pour lesquels la physique (souvent complexe) est rarement prise en compte. Les difficultés liées à la vitesse de sollicitation se traduisent d'une part au niveau expérimental par des essais souvent indirects et globaux (e.g., impact de plaques, essais aux barres de Hopkinson-Kolsky) et d'autre part au niveau numérique où tous les problèmes liés à l'intégration d'une loi de comportement et de rupture ne sont pas encore résolus. La formulation d'une loi de comportement doit donc prendre en compte, dès l'origine, les difficultés liées à l'identification des paramètres représentatifs du matériau et les possibilités d'implantation en fonction du code envisagé.

Des avancées significatives ont été observées ces dernières années, notamment en France dont on notera l'existence passée (et non pérennisée, pour l'instant) du GDR 972 « Impact-matériaux » [76] et d'un groupe spécifiquement lié aux problèmes dynamiques dans le cadre du Réseau «GEO»[26]. Sous l'impulsion de deux associations DYMAT et MECAMAT, membres sectoriels de l'AFM, un groupe de travail «MECADYMAT » a été créé. Ce groupe a pour but d'établir un lieu d'échange informel et convivial entre industriels et universitaires autour de la modélisation du comportement et de la rupture des matériaux sous sollicitations dynamiques. Les problèmes traités concernent la formulation, l'identification et l'implantation de lois de comportement fiables de matériaux sous sollicitations dynamiques; l'objectif étant de modéliser les dégradations (e.g., endommagement) et la ruine (e.g., rupture) de ces matériaux. Les premières journées se sont 
tenues à l'ENSMA de Poitiers au mois d'avril 2000. Les débats organisés à la fin de chaque session, et la synthèse finale, peuvent se rassembler autour de quelques lignes de force :

- nécessité d'une méthodologie qui intègre les aspects expérimentaux, théoriques et numériques sur le même plan ;

- manque de méthodes expérimentales fiables ;

- besoin de lois de comportement simples, sûres, et facilement identifiables ;

- nécessité, dans de nombreux cas, d'une meilleure prise en compte des processus physiques de déformation et/ou de dégradation ;

- manque crucial de description et, plus généralement, de connaissances des processus de rupture ;

- besoin de prise en compte de la notion de variabilité et des aspects microstructuraux des matériaux ;

- fort déficit de lois de comportement fiables pour les polymères, mousses et composites ;

- besoin crucial d'outils numériques adéquats et fiables, absence presque totale d'outils adaptés à la description de la rupture ;

- peu de modèles numériques disponibles pour les assemblages.

Les champs de recherche et de développement représentent sans aucun doute un véritable défi pour la communauté scientifique et conduiront également à des applications industrielles audacieuses dans des domaines de plus en plus étendus. 


\section{Références bibliographiques}

1. Allix O., Guédra-Degeorges D., Guinard S., Vinet A., Analyse de la tenue aux impacts à faible vitesse et faible énergie des stratifiés composites par la mécanique de l'endommagement, Méc. Ind. 1 (2000) 27-35.

2. Hild F., Rota L., Comportement et rupture de matériaux sous sollicitations dynamiques - Une esquisse d'état de l'art - Objectifs du groupe de travail, communication lors des premières journées du groupe de travail MECADYMAT, ENSMA Poitiers (2000).

3. Deletombe E., Travaux expérimentaux et numériques liés aux applications de crash aéronautique, communication lors des premières journées du groupe de travail MECADYMAT, ENSMA Poitiers (2000).

4. Lory P., Lois de comportement et critères de rupture en dynamique pour applications automobiles, communication lors des premières journées du groupe de travail MECADYMAT, ENSMA Poitiers (2000).

5. Tran P., Rupture des matériaux plastiques, communication lors des premières journées du groupe de travail MECADYMAT, ENSMA Poitiers (2000).

6. Cottenot C.E., Quelques exemples de besoins en modélisation de la ruine des matériaux en dynamique : applications aux blindages et aux têtes militaires, communication lors des premières journées du groupe de travail MECADYMAT, ENSMA Poitiers (2000).

7. de Resseguier T., Boustie M., Caractérisation et modélisation de la réponse des matériaux au choc laser, communication lors des premières journées du groupe de travail MECADYMAT, ENSMA Poitiers (2000). 
8. Malaise F., Lois de comportement et d'endommagement sous choc de matériaux composites et de poreux basse densité, communication lors des premières journées du groupe de travail MECADYMAT, ENSMA Poitiers (2000).

9. Lemaitre J., Chaboche J.-L., Mécanique des matériaux solides, Dunod, Paris, 1985.

10. Bui H.D., Mécanique de la rupture fragile, Masson, Paris, 1978.

11. Labbens R.C., Introduction à la mécanique de la rupture, Pluralis, Paris, 1980.

12. Bathias C., Baïlon J.-P., La fatigue des métaux et des structures, Presses de l'Université de Montréal, Montréal, Canada, 1980.

13. Denoual C., Hild F., A damage model for the dynamic fragmentation of brittle solids, Comp. Meth. Appl. Mech. Engrg. 183 (2000) 247-258.

14. Cagnoux J., Déformation et ruine d'un verre pyrex soumis à un choc intense : étude expérimentale et modélisation du comportement, Thèse de Doctorat ès Sciences Physiques, Université de Poitiers, 1985.

15. Hopkinson D., A method of measuring the pressure produced in the detonation of high explosives or by the impact of bullets, Phil. Trans. Roy. Soc. A 213 (1914) 437-452.

16. Kolsky H., An investigation of the mechanical properties of materials at very high rates of loading, Proc. Phys. Soc. London, 62b (1949) 676-700.

17. Gary G., Zhao H., Étude expérimentale du comportement dynamique des matériaux, Méc. Ind. 1 (2000) 15-26.

18. Gary G., Klepaczko J., Zhao H., Corrections for wave dispersion and analysis of small strain with split Hopkinson bars, Actes Int. Conf. Imp. Eng., Sendai, Japon, 1989.

19. Rota L., Application de méthodes inverses au dépouillement de l'essai aux barres de Hopkinson, Thèse de Doctorat de l'Ecole Polytechnique, 1997. 
20. Meyers M.A., Dynamic behavior of materials, John Wiley and Sons, New York, USA, 1994.

21. Cosculluela A., Plasticité, endommagements et rupture des alumines sous sollicitations dynamiques triaxiales: influence de la taille des grains, Thèse de Doctorat de l’Université de Bordeaux I, 1992.

22. Cagnoux J., Chartagnac P., Héreil P.-L., Perez M., Lagrangian analysis-Modern tool of the dynamics of solids, Ann. Phys. Fr. 12 (1987) 451-524.

23. Farré J., Étude du comportement dynamique des matériaux au CEA Valduc : démarche pour le développement et l'évaluation de modèles de comportement, communication lors des premières journées du groupe de travail MECADYMAT, ENSMA Poitiers, 2000.

24. Chen D.Y., Gupta Y.M., Miles M.H., Quasistatic experiments to determine material constants for the piezoresistance foils used in shock wave experiments, J. Appl. Phys. 55 (1984) 3984-3993.

25. Wong M.K.W., Experiments and analysis to understand the response of lateral piezoresistance gauges under dynamic loading, $\mathrm{PhD}$ Dissertation, Washington State University, USA, 1991.

26. Bailly P., Kéryvin V., Dynamique de la rupture du béton et son comportement post-pic : un point clé pour décrire et prévenir la rupture d'une structure et ses conséquences, communication lors des premières journées du groupe de travail MECADYMAT, ENSMA Poitiers, 2000.

27. Mason J.J., Rosakis A.J., Ravichandran G., On the strain and strain rate dependence of the fraction of plastic work converted to heat: an experimental study using high speed infrared detectors and the Kolsky bar, Mech. Mat. 7 (1994) 135-145. 
28. Léal B., Application de la pyrométrie optique à la mesure de la température des produits de réaction d'explosifs condensés en régime d'amorçage et de détonation, Thèse de Doctorat de l’Université de Poitiers \& ENSMA, 1998.

29. Trumel H., Un modèle viscoélastique-viscoplastique pour un composite particulaire. Application à un problème de pénétration dynamique, Thèse de doctorat de l'Université de Poitiers \& ENSMA, 1996.

30. Strassburger E., Senf H., Experimental investigations of wave and fracture phenomena in impacted ceramics and glasses, Report ARL-CR-214, 1995.

31. Woodward R.L., Burman N.M., Baxter B.J., An experimental and analytical study of the Taylor impact test, Int. J. Impact Eng. 15 (1994) 407-416.

32. Petit J., Alexeev Y.A., Ananiev S.P., Kazeev M.N., The electromagnetic cylindrical compression : a tool to test behaviour modelling under large strain at high strain-rate, J. Phys. IV France (1997) 109-114.

33. Bertin-Mourot T., Denoual C., Dehors G., Louvigné P.-F., Thomas T., High speed photography of moiré fringes - Application to ceramics under impact. J. Phys. IV France (1997) 311-316.

34. Rastogi P.K., Photomechanics, Topics in Appl. Phys., 77, Springer Verlag, Berlin (Allemagne Fédérale), 2000.

35. Erlich D.C., Chartagnac P., Determination of dynamic flow curve of metals at ambient and elevated temperatures by rod impact techniques, J. Phys. France 46 (1985) 455-462.

36. Riou P., Contribution à l'étude de l'endommagement du carbure de silicium lors d'un impact de basse énergie : application aux blindages, Thèse de Doctorat de l'Ecole Nationale Supérieure des Mines de Paris, 1996. 
37. Mercier S., Molinari A., Steady-state shear band propagation under dynamic conditions, J. Mech. Phys. Solids 46 (1998) 1463-1495.

38. Curran D.R., Seaman L., Murri W.J., Dynamic failure of solids, Phys. Rep. 147 (1985) 253-388.

39. Maigre H., Contribution théorique à l'identification des grandeurs caractéristiques en mécanique de la rupture dynamique, Thèse de Doctorat de l'Ecole polytechnique, 1990.

40. Dos Martires N., Mise au point d'une procédure de détermination de la ténacité sous sollicitation dynamique d'un matériau ductile, Thèse de Doctorat de l'Ecole Polytechnique, 1999.

41. Fanget A., Problématique numérique dans la prévision du comportement et de la rupture dynamique, communication lors des premières journées du groupe de travail MECADYMAT, ENSMA Poitiers, 2000.

42. Dhatt G., Touzot G., Une présentation de la méthode des éléments finis, Maloine, Paris, 1981.

43. Hilber H.M., Hughes T.J.R., Taylor R.L., Collocation, Dissipation and "Overshoot" for Time Integration Schemes in structural Dynamics, Earthquake and Structural Dynamics $6(1978) 99-117$.

44. Wilkinson J.H., Reinsch C., Linear Algebra, in : Handbook for Automatic Computation, II, Springer Verlag, New York, USA, 1971.

45. Fried I., Numerical Solution of Differential Equations, Academic Press, New York, USA, 1979.

46. Donea J., Arbitrary Lagrangian-Eulerian Finite Element Method, in : Computational Methods for Transient Analysis, Belytschko T., Hughes T.J.R. (éd.), North-Holland, Amsterdam, Hollande, 1983, pp. 473-516, 1983. 
47. Johnson G.R., Cook W.H., Fracture characteristics of three metals subjected to various strains, strain rates, temperatures and pressures, Engrg. Fract. Mech. 21 (1985) 31-48.

48. Descombes C., Traitement des contacts avec frottement dans un logiciel explicite 3D de dynamique rapide, modèles numériques en configuration élastoplastique $1 \mathrm{D}$, Thèse de l'Université de Bordeaux I, 1997.

49. Rio G., Grolleau V., Couty N., Manach P.-Y., Simulation numérique par éléments finis de phénomènes de choc : modélisation en dynamique incluant un comportement d'élasto-visco-hystérésis volumique et coque. Application au souffle aérien et à l'impact de plaques, communication lors des premières journées du groupe de travail MECADYMAT, ENSMA Poitiers, 2000.

50. Rebuffet O., Modélisation du comportement au crash des structures aluminium pour les applications automobiles, communication lors des premières journées du groupe de travail MECADYMAT, ENSMA Poitiers, 2000.

51. Pluvinage G., Critère de rupture du verre soumis à l'impact, communication lors des premières journées du groupe de travail MECADYMAT, ENSMA Poitiers, 2000.

52. Gurson A.L., Continuum theory of ductile rupture by void nucleation and growth Part I: Yield criteria and flow rules for porous ductile media, J. Eng. Mat. Tech. 99 (1976) 215.

53. Rauscher E., Modélisation de l'endommagement du béton : besoins industriels, communication lors des premières journées du groupe de travail MECADYMAT, ENSMA Poitiers, 2000.

54. Bonnan S., Modélisation pour le crash, communication lors des premières journées du groupe de travail MECADYMAT, ENSMA Poitiers, 2000. 
55. Deprince X., Petitpas E., Modélisation de la ruine de matériaux métalliques: application à la perforation dynamique, communication lors des premières journées du groupe de travail MECADYMAT, ENSMA Poitiers, 2000.

56. Mazeau C., Beylat L., Longère P., Louvigné P.-F., On the quantitative evaluation of adiabatic shear banding sensitivity of various titanium alloys, J. Phys. IV France (1997) 429-434.

57. Beremin F. M., A local criterion for cleavage fracture of a nuclear pressure vessel steel, Metallurgical Transactions 14A (1983) 2277-2287.

58. Needleman A., Tvergaard V., An analysis of dynamic, ductile crack growth in a double edge cracked specimen, Int. J. Fract. 49 (1991) 41-61.

59. Tong W., Ravichandran G., Inertial effects on void growth in porous viscoplastic materials, J. Appl. Mech. 62 (1995) 633-639.

60. Wang Z.P., Jiang Q., A yield criterion for porous ductile media at high strain-rate, J. Appl. Mech. 64 (1997) 503-509.

61. Baladi G.Y., B. Rohani, Development of an elastic-viscoplastic constitutive relationship for earth materials, in : Mechanics of Engineering Materials, Desai C.S., Gallagher R.H. (éd.), John Wiley and Sons, New York, USA, 1984.

62. Darve F., Hicher P.-Y., Reynouard J.-M. (coordonateurs), Mécanique des géomatériaux, série «Etudes en mécanique des matériaux et des structures », Hermès, Paris, vol. 2, 1995, pp. 39-102.

63. Johnson G.R., Holmquist T.J., An improved computational constitutive model for brittle materials, High Press. Sci. Tech. 2 (1992) 981-984.

64. Seaman L., Curran D.R., Shockey D.A., Computational models for ductile and brittle fracture, J. Appl. Phys. 47 (1976) 4814-4826. 
65. Dienes J.K., A statistical theory of fragmentation processes, Mech. Mat. 4 (1984) 325335.

66. Grady D.E., The spall strength of condensed matter, J. Mech. Phys. Solids 36 (1988) 353-384.

67. O’Dowd N.P., Knauss et W.G., Time dependent large principal deformation of polymers, J. Mech. Phys. Solids 43 (1995) 771-792.

68. Govindjee S., Reese S., A presentation and comparison of two large deformation viscoelasticity models, J. Engng. Mat. Technol., 119 (1997) 251-255.

69. Boyce M.C., Parks D.M., Argon A.S., Large inelastic deformation of glassy polymers. Part I : Rate dependent constutive model, Mech. Mat. 7 (1988) 15-33.

70. Bonnet N., Etude du comportement sous sollicitations intenses d'élastomères Application au cas du blindage réactif, Thèse de Doctorat de l'Ecole Nationale Supérieure des Arts et Métiers, 1998.

71. Simo J.C., On a fully three-dimensional finite-strain viscoelastic damage model: formulation and computational aspects, Comp. Meth. Appl. Mech. Engrg. 60 (1987) 153-173.

72. Ha K., Schapery R.A., A three-dimensional viscoelastic constitutive model for particulate composites with growing damage and its experimental validation, Int. J. Solids Struct. 35 (1998) 3497-3517.

73. Gibson L., Ashby M.F., Cellular Solids. Structure and Properties, $2^{\text {nd }}$ edition, Pergamon Press, New York, USA, 1997.

74. Ladevèze P., About a damage mechanics approach, in : Mechanics and Mechanisms of damage in composites and multimaterials, Baptiste D. (éd.), MEP, London, UK, 1991, pp. 119-142. 
75. Sabina F.J., Willis J.R., Self-consistent analysis of waves in a polycrystalline medium, Eur. J. Mech., A/Solids 12 (1993) 265-275.

76. Lataillade J.-L., Louvigné P.-F., Rapport scientifique du Groupement de Recherche 972 CNRS-DRET "Impact-Matériaux" 1994-1995, 1996. 


\section{Légende des figures}

Figure 1: classification des phénomènes dynamiques en fonction d'un temps caractéristique de variation de chargement.

Figure 2: démarche générale de construction d'un modèle de comportement, d'endommagement ou de rupture.

Figure 3: montage d'essai de compression dynamique de barres de Hopkinson.

Figure 4: microfissuration d'un carbure de silicium $2 \mu$ s et $3 \mu$ s après impact d'un barreau métallique à une vitesse de 203 m/s (d'après Riou [36]).

Figure 5: cisaillement adiabatique dans un alliages de titane (TA6V).

(source DGA/DCE/Centre Technique d'Arcueil)

Figure 6: comportement de deux alliages de titane face à un impact

(source DGA/DCE/Centre Technique d'Arcueil)

a-TA6V - mécanisme "ductile"

b-ßCEZ - mécanisme "fragile" avec écaillage

Figure 7: différentes démarches en mécanique de la déformation, de la dégradation et de la rupture sous sollicitations dynamiques.

Figure 8: a-exemple de franges de moiré $2 \mu$ s après l'impact d'un carreau de carbure de silicium par un barreau métallique à une vitesse de $330 \mathrm{~m} / \mathrm{s}$.

b-champ de déformation principale maximale.

c-champ de déformation principale minimale.

Figure 9: mousse et nid d'abeille en alliage d'aluminium. 


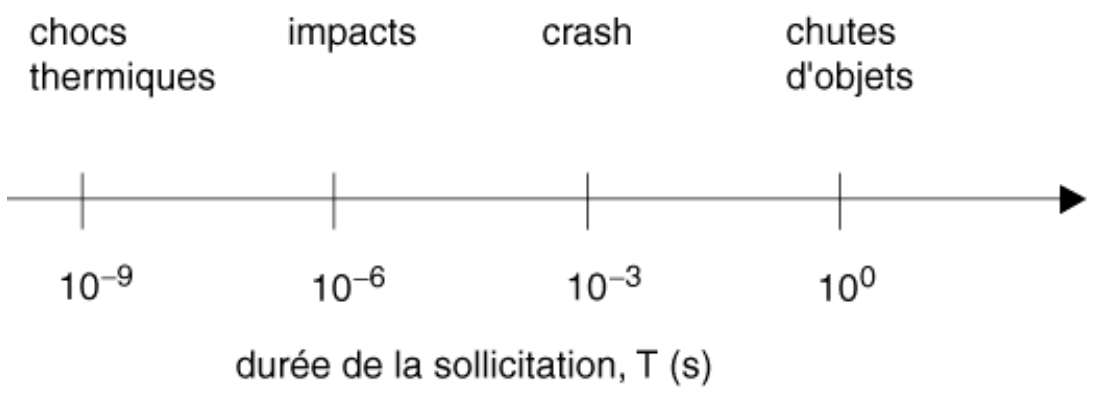

Figure 1 : classification des phénomènes dynamiques en fonction d'un temps caractéristique de variation de chargement. 


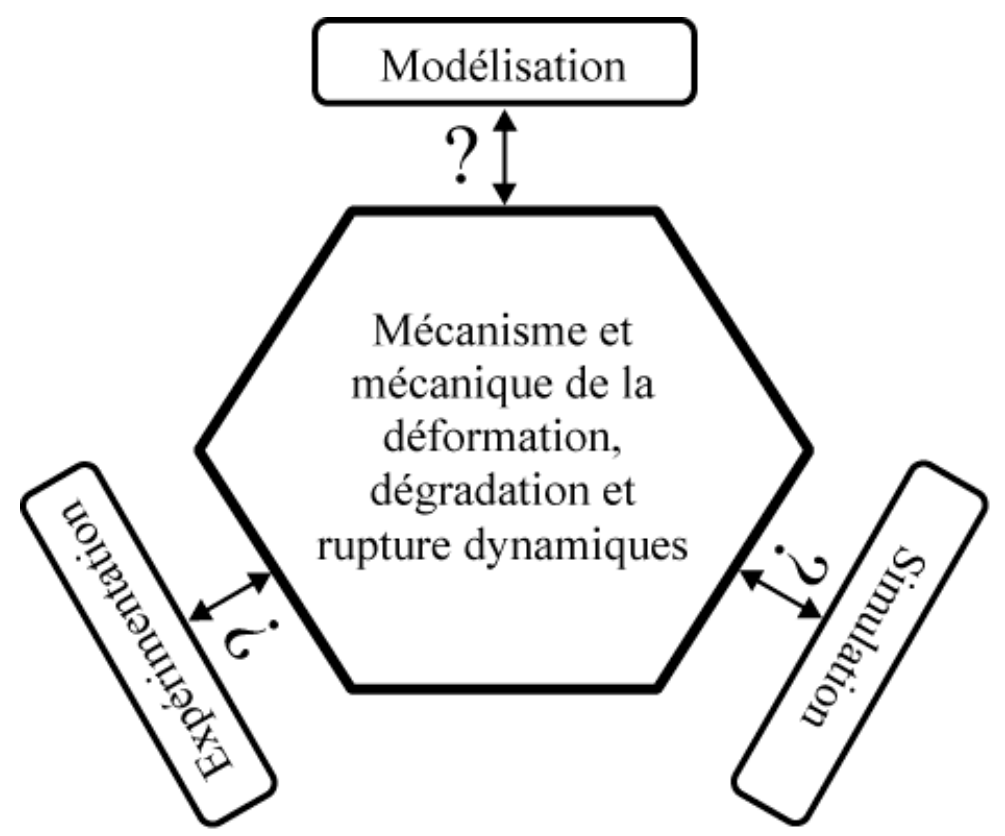

Figure 2 : démarche générale de construction d'un modèle de comportement, d'endommagement ou de rupture. 


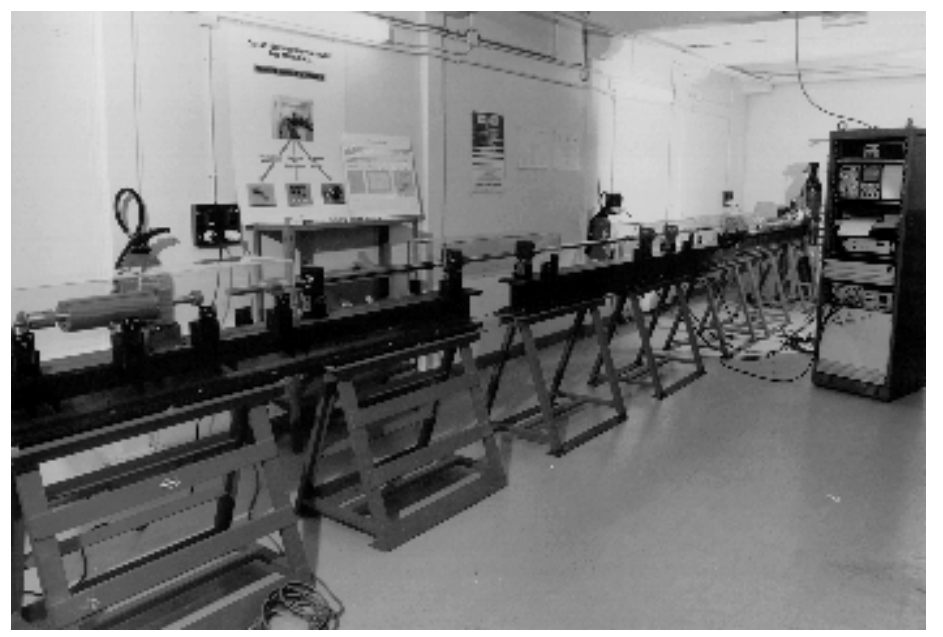

Figure 3 : montage d'essai de compression dynamique de barres de Hopkinson. 


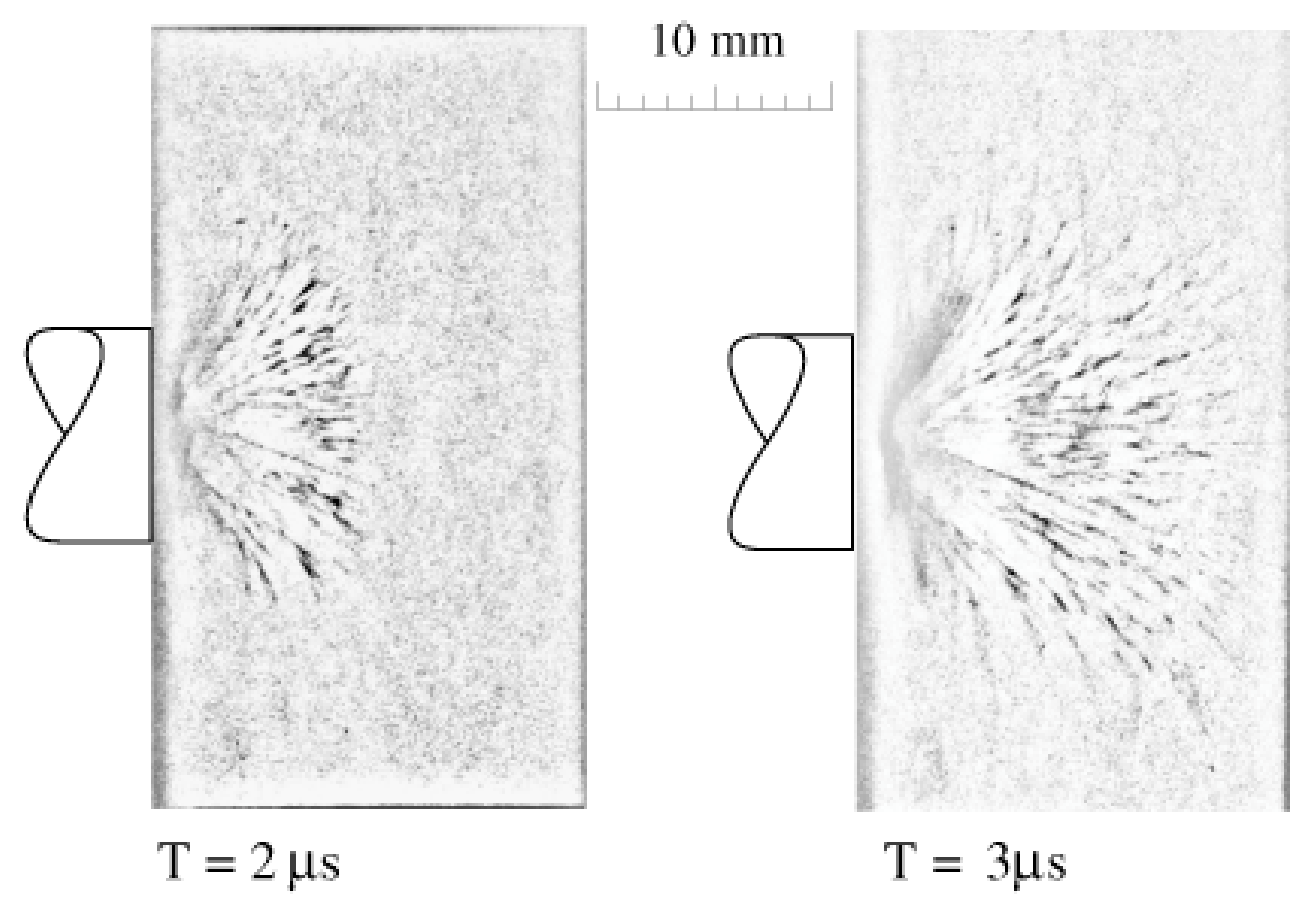

Figure $4:$ microfissuration d'un carbure de silicium $2 \mu$ s et $3 \mu$ s après impact d'un barreau métallique à une vitesse de $203 \mathrm{~m} / \mathrm{s}$ (d'après Riou [36]). 


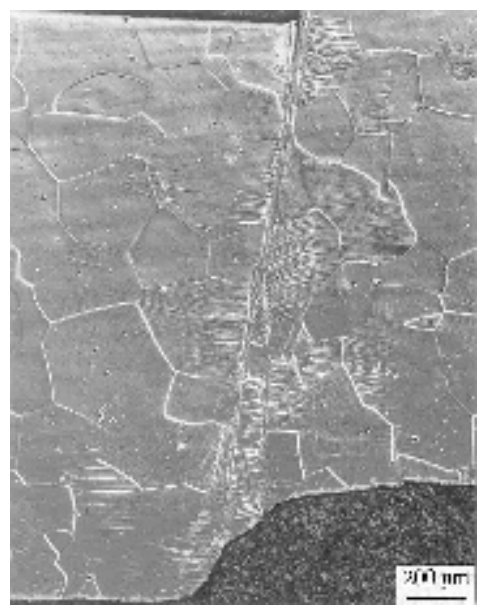

Figure 5 : cisaillement adiabatique dans un alliages de titane (TA6V) (source DGA/DCE/Centre Technique d'Arcueil) 

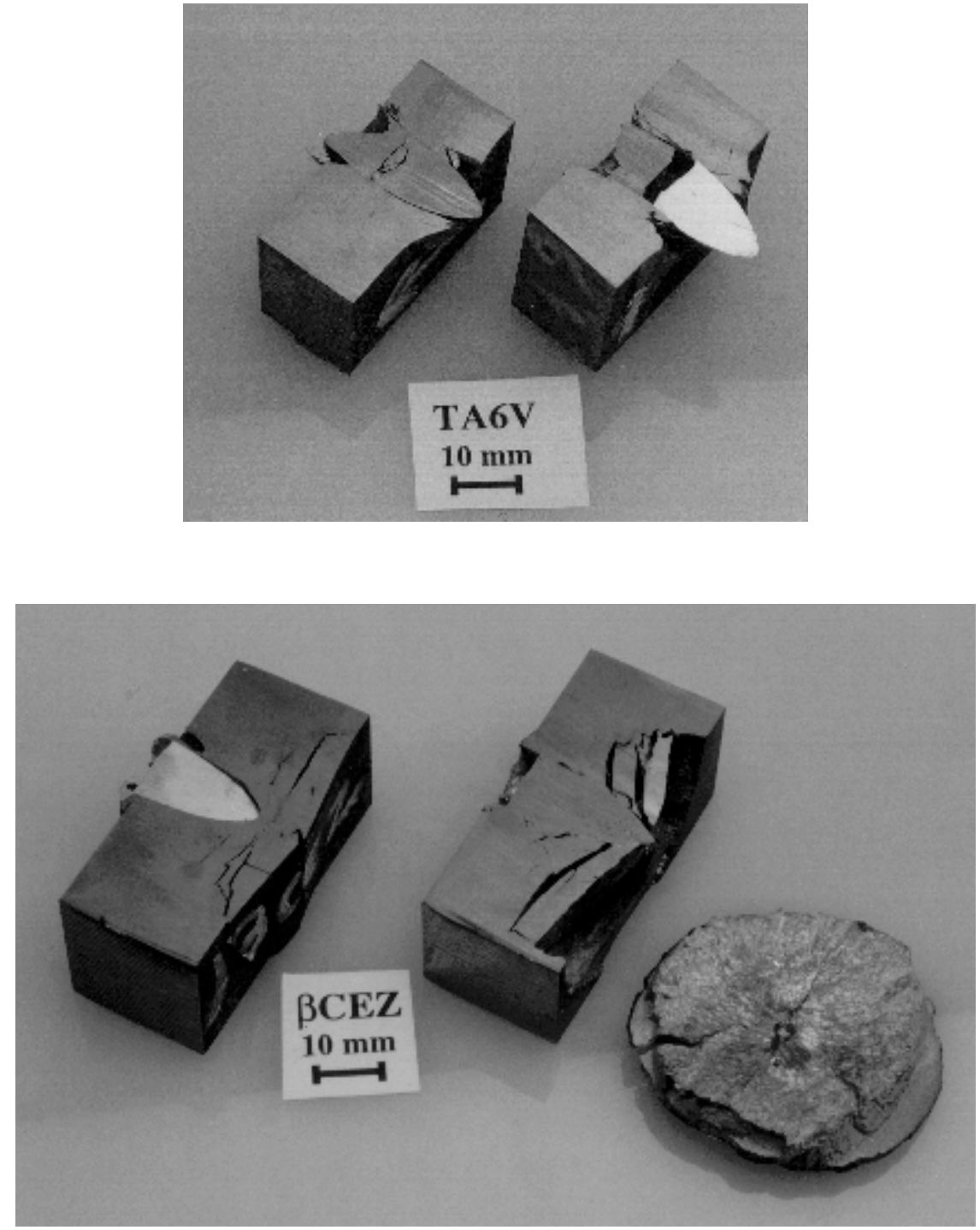

Figure $6:$ comportement de deux alliages de titane face à un impact (source DGA/DCE/Centre Technique d'Arcueil) a-TA6V - mécanisme "ductile". b- $\beta C E Z$ - mécanisme "fragile" avec écaillage. 


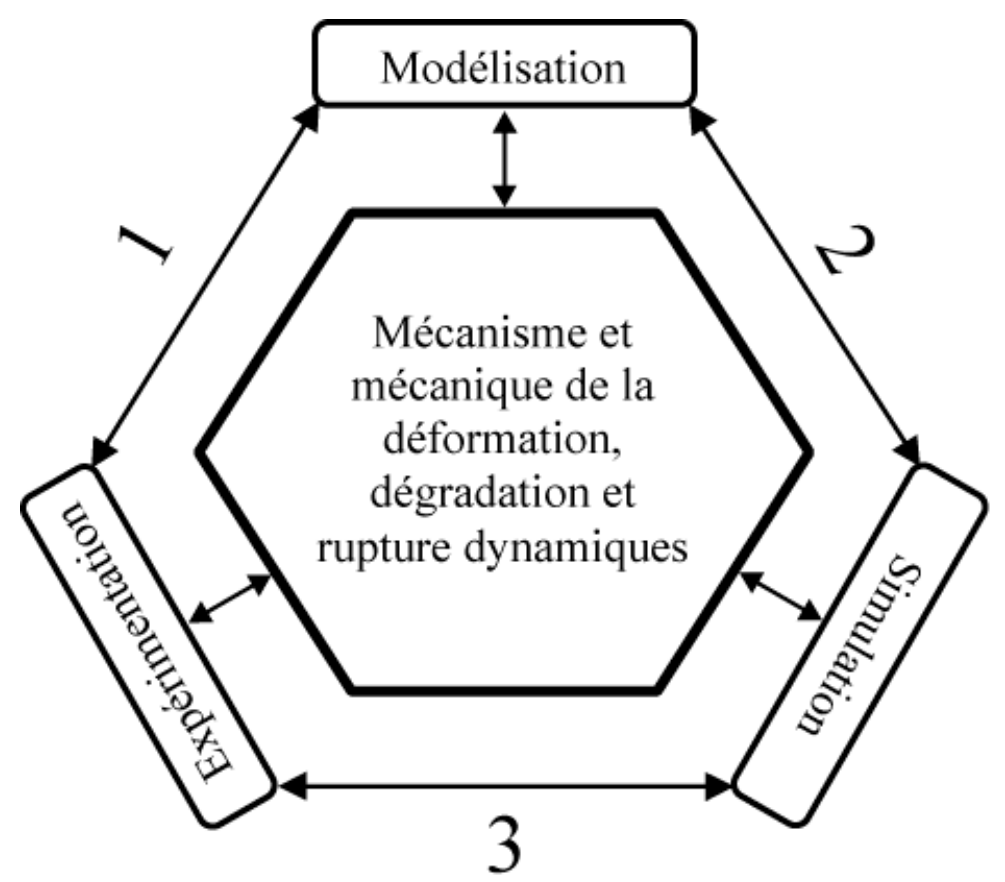

Figure 7 : différentes démarches en mécanique de la déformation, de la dégradation et de la rupture sous sollicitations dynamiques. 

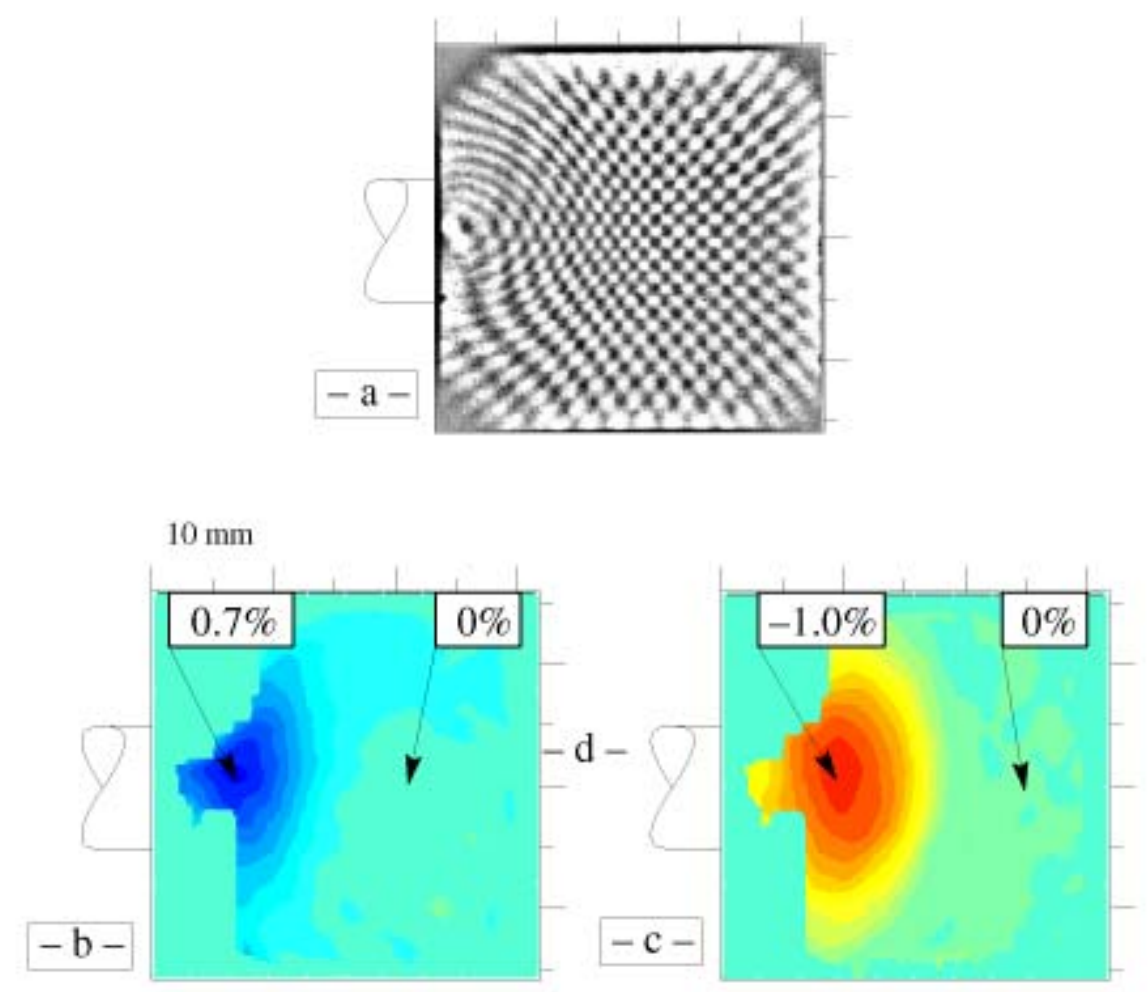

Figure 8 : a-exemple de franges de moiré $2 \mu$ s après l'impact d'un carreau de carbure de silicium par un barreau métallique à une vitesse de $330 \mathrm{~m} / \mathrm{s}$.

b-champ de déformation principale maximale.

c-champ de déformation principale minimale. 

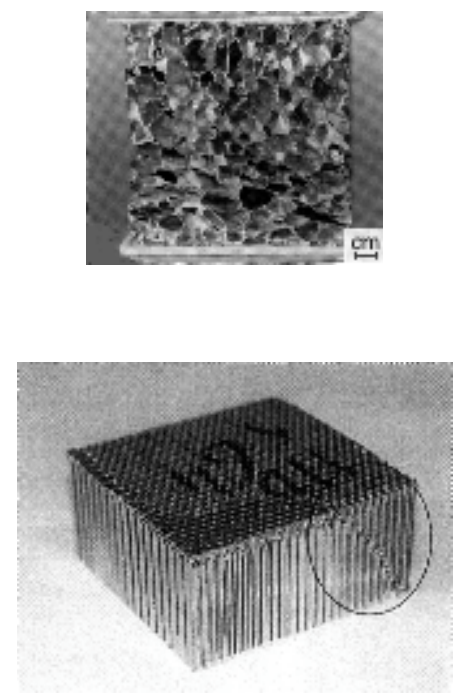

Figure 9 : mousse et nid d'abeille en alliage d'aluminium. 